\title{
Use of MSC in the Treatment of the Congenital Pseudoarthrosis in Children
}

\author{
Marina Magnani*, Costantina Racano, Caterina Novella Abati, Donatella Granchi, \\ Valentina de Vescovi, Stefano Stilli \\ Istituto Ortopedico Rizzoli (IRCCS), Bologna, Italy \\ Email: marina.magnani@ior.it
}

Received 23 September 2014; revised 20 October 2014; accepted 15 November 2014

Academic Editor: Vahit Ozmen, Istanbul University, Turkey

Copyright (C) 2014 by authors and Scientific Research Publishing Inc.

This work is licensed under the Creative Commons Attribution International License (CC BY).

http://creativecommons.org/licenses/by/4.0/

(c) (i) Open Access

\begin{abstract}
Congenital pseudoatrhrosis of the tibia is one of the most frustrating conditions encountered in paediatric orthopaedic surgery because of the difficulty in achieving healing; There are different methods of treatment the most commonly used are: External fixator according Ilizarov's technique, vascularised fibular grafting, bone grafting with intramedullary fixation, Boyd's double bone grafting and also after several operation go bad and a significant shortening of the leg even the amputation has to be considered [1] [2]. Numerous treatment options have been explored with several degree of success. In this paper we show a combinated surgical technique using autogenous mesenchymal stem cells (MSC) by precursor of osteogenic differensation at the same time as adjuvant to the surgical stabilization by an external fixator or an intramedullary nailing. We used these combined technique in tibia congenital pseudoarthrosis with and without neurofibromatosis in children. In fact Bone Marrow has been shown to contain a population of rare mesenchymal stem cells that are capable of forming bone, cartilage and other connective tissues enhancing bone repair and regeneration.
\end{abstract}

\section{Keywords}

Pseudoarthrosis, Mesenchimal Stem Cells, Children

\section{Introduction}

Neurofibromatosis Type I or Recklinghausen disease, is one of the most common congenital disease and is one

*Corresponding author. 
of the most common congenital diseases with a prevalence of one in $3000-4000$.

The diagnosis is established clinically by detecting the characteristic phenotypic manifestations such as cafe'aulait spots, axillary and inguinal freckling, neurofibromas, optic gliomas, Lisch nodules and distinctive osseous lesions. The latter are believed to be related to a deficient osteoblast function with enhanced osteoclast activity and survival occurring in approximately $2 \%$ - 3\% of children suffering from neurofibromatosis type I.

The most affected bone is the tibial diaphysis and the displastic lesion at the radiological appearance can look like an anterior bowing, or a sclerotic lesion or a fracture.

The congenital pseudarthrosis of the tibia (CPT) with or without NF1 is one of the most complex and disabling paediatric skeletal diseases. Conservative treatments are bracing of the affected limb continued until the end of skeletal growth [3]. Surgical treatment includes several procedures which are resection of the non-union and reconstruction with either autologous non-vascolarised bone grafting and intramedullary fixation or one stage shortening followed by an ilizarov external fixator to perform lengthening [1]. All these procedure have frequently unsatisfactory results because of the need for several operations for recalcitrant non-union, residual deformity and limb-length discrepancies.

Recently the use of autologous MSC has garnered great interest, because the biological properties of MSCs candidate them as a suitable source of osteogenic precursor for bone repair and regeneration, both in cell therapy and tissue engineering applications [4]-[6].

Surgical treatment of pseudarthrotic lesions could benefit of regenerative medicine, which provides a biological approach to consolidation, based on the use of cells and growth factors. These elements could lead to tissutal healing and consolidation, in cases where traditional techniques fail. This work describes an innovative technique, resulting from a multidisciplinary collaboration, and the clinical and radiographic features of the different types of pseudarthrosis, both at presentation and at the time of the definitive surgical treatment.

\section{Material and Methods}

We presented 13 patients affected of tibial congenital pesudoarthrosis: 10 male and 3 female. The average age at the time of operation was 7.7 years (min 9 months, max 14 years) 11 of these 13 patients had also a fibula's pseudoarthrosis.

All had unilateral involvement 5 patients had neurofibromatosis ( 1 female and 4 male) 2 patients had an osteofibrous dysplasia and 6 patients had a congenital idiopathic pseudoartrosis (Table 1).

According to the Crawford classification (Figure 1) we define the dysplasic lesion as the radiographic appearance at the diagnosis and we considered:

Type 1 anterolateral bowing with medullary sclerosis.

Type II sclerotic lesion with narrowing of the cortical diameter.

Type III a cystic lesion.

Type IV frank pseudoarthrosis (Figures 1(a)-(d)).

\begin{tabular}{|c|c|c|c|c|c|c|c|c|c|}
\hline $\mathbf{N}$ & $\mathbf{P Z}$ & $\mathbf{S}$ & AGE & SIDE & NF & CALFFISICATION & FIBULA & CONGENITAL & PROCEDURE \\
\hline 1 & BG & $\mathrm{F}$ & $14 \mathrm{y}$ & LEFT & NO & 1 & YES & YES & INTRAMEDULLARY NAILING \\
\hline 2 & FJ & $\mathrm{F}$ & $13 \mathrm{y}$ & RIGHT & NO & 3 & NO & NO & INTRAMEDULLARY NAILING \\
\hline 3 & MMA & M & 3 y & LEFT & NO & 4 & YES & YES & INTRAMEDULLARY NAILING \\
\hline 4 & MF & M & $6 y$ & LEFT & YES & 4 & YES & YES & INTRAMEDULLARY NAILING \\
\hline 5 & MMA & M & $14 \mathrm{y}$ & LEFT & YES & 4 & YES & YES & INTRAMEDULLARY NAILING \\
\hline 6 & MEP & $\mathrm{F}$ & $9 \mathrm{~m}$ & LEFT & YES & 4 & YES & YES & INTRAMEDULLARY NAILING \\
\hline 7 & $\mathrm{PA}$ & M & $13 \mathrm{y}$ & RIGHT & NO & 1 & YES & YES & INTRAMEDULLARY NAILING \\
\hline 8 & $\mathrm{PD}$ & M & 3 y & RIGHT & YES & 1 & YES & YES & INTRAMEDULLARY NAILING \\
\hline 9 & $\mathrm{RAD}$ & M & $14 \mathrm{y}$ & RIGHT & YES & 4 & NO & NO & EXTERNAL CIRCULAR FIXATOR \\
\hline 10 & RS & M & $8 \mathrm{y}$ & RIGHT & NO & 4 & YES & NO & NTRAMEDULLARY NAILING \\
\hline 11 & VE & M & $13 \mathrm{y}$ & RIGHT & YES & 4 & YES & NO & INTRAMEDULLARY NAILING \\
\hline
\end{tabular}

Patients affected by pseudoartrosis of the tibia classification of the lesions by Catteral lassification, age of the patients, associations with NF1 or not, associated lesions of the fibula or not, Treatment with nails or with external fixator. 


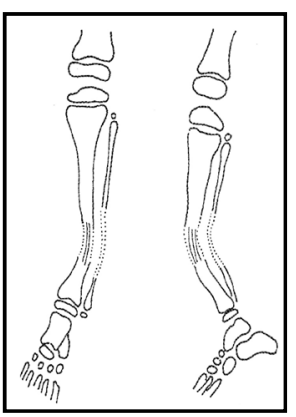

(a)

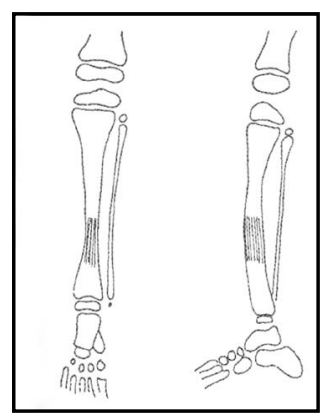

(b)

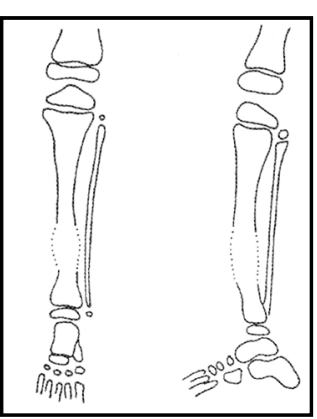

(c)

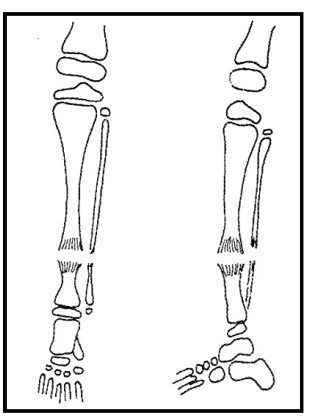

(d)

Figure 1. (a) Dysplastic form; (b) Sclerotic form; (c) Cystic form; (d) Fractured form.

We had 5 types I, 0 type II, 2 type III, 6 type IV.

5 patients had no a previous surgery, 8 patients had at least one unsuccessful surgical procedure all the patients had established pseudarthrosis.

The surgical treatment consists in two steps: After you have collected from parents of the patients.

Regular informed consent to participate in the study and to undergo treatment combined surgical and with the use of MSC.

The day before surgery we take from the patient $100 \mathrm{ml}$ of venous blood from which derive a concentration of platelet and grow factors to use the day of surgery and to put in to the lyophilized bone graft.

The first step of the surgery is the Bone marrow aspiration from the iliac crest of the patients by an anterior or posterior approach to the iliac crest and in general anaesthesia (from 20 to $60 \mathrm{ml}$ ).

The bone marrow is centrifuged, and $50 \mathrm{ml}$ buffy coat is obtained (mean of $24.35 \times 10^{3} \pm$ bone marrow cells) per microliter [2] [7].

The second step is the surgical stabilization; we used two types of procedures.

In 12 patients the tibial pseudoarthrosis was resected extraperiosteally proximally and distally to bleeding bone. The medullar canal was opened with a drill. The tibia was shortened and internally fixed with a rush wire or a $\mathrm{k}$ wire transfixed also the ankle and subtalar joints. We also performed a fibular distal osteotomy if the fibula is intact, in either case we don't fix the fibula pseudoartrhosis to low impaction to the tibial resection site.

After this operation the patient wore a leg cast for one month and then a brace until the bone consolidation.

In 1 patient we used an external circular fixator according the ilizarov technique to correct the axial misalignment and leg shortening at the same time. First, excision of the fibrous tissue at the pseudarthrosis site, then the ilizarov fixator was installed with two whole rings above the non-union site and one bellow, a compression was applied on the bone defect and a distraction at the proximal metafiseal site by a compactotomy to allow the bone segmental transportation. Distraction speed for bone transportation procedures was $1 \mathrm{~mm} /$ day.

The surgical time ends by the implant of a packed of lyophilised bone graft (obtained from the bone bank) with autologous stromal cells (obtained from the bone marrow aspiration) and growth factors (obtained from the platelet of the patients by venous blood the day before surgery) circumferentially around the bone lesion site. Periosteum (when it is possible) is also placed around the bone lesion site or a muscle.

After the transported segment had bridged the gap, end to end compression was applied; after bone union was achieved the ilizarov fixator was removed and applied a long leg cast for one month and then a protecting or thesis for three months.

At one month, three months, six mounts and one year after surgery, the patients underwent a clinical and a radiographic evaluation.

\section{Results (Table 2)}

At the final follow-up 4 years ( 4 years, range 1 month to 4 years) the patients were 11 we lost 2 patients who had changed hospital. We have revised the 11 patients clinically observing the range of motion of the anke and the knee, the presence of pain, the skin and the stability of the leg. We take an anteroposterior and a lateral xr of the leg, at the least follow-up we see the healing of the pseudarthrosis (no radiological evidence of bone lesions) and presence of union in three patients of the 11: two of these were treated with intramedullary nailing and one patient was treated with the ilizarov external fixator. 
Case one: Patient male three years old at the time of surgery with a congenital tibia pseudoarthrosis without NF1, he had no previous surgery, and he was treated with intramedullary nailing and MSCs. The pseudorthrosis healing after six months and after nine months from surgery we have seen the nail fracture bellow the site of bone lesion that was still good (Figures 2(a)-(g)).

Table 2. Results.

\begin{tabular}{ccccc}
\hline No & PZ & Complication & Healing & Last F.U. \\
\hline 1 & B.G. & No & $29 \mathrm{~m}$ \\
2 & F.J. & No & $31 \mathrm{~m}$ \\
3 & M.M.A. & No & $6 \mathrm{~m}$ & $37 \mathrm{~m}$ \\
4 & M.F. & Deep infection & $5 \mathrm{~m}$ \\
5 & M.M.A. & No & $32 \mathrm{~m}$ \\
6 & M.E.P. & No & & $48 \mathrm{~m}$ \\
7 & P.A. & No & & $15 \mathrm{~m}$ \\
8 & P.D. & No & & $41 \mathrm{~m}$ \\
9 & R.A.D. & New pseudoarthrosis (proximal metaphsis) during lengthening & $7 \mathrm{~m}$ & \\
10 & R.S. & Yes & & $33 \mathrm{~m}$ \\
11 & V.F. & No & & $19 \mathrm{~m}$ \\
\hline
\end{tabular}

Patients treated and their follow up. Tow complications: one deep infection and one new pseudoatrhosis. Only three patiens healed.

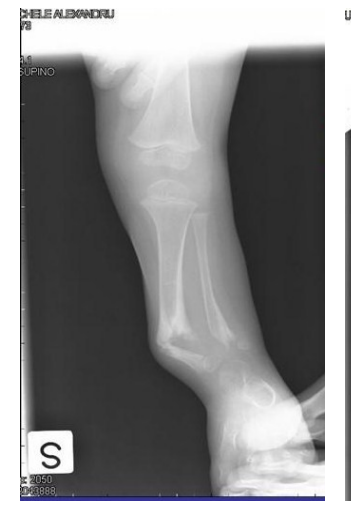

(a)

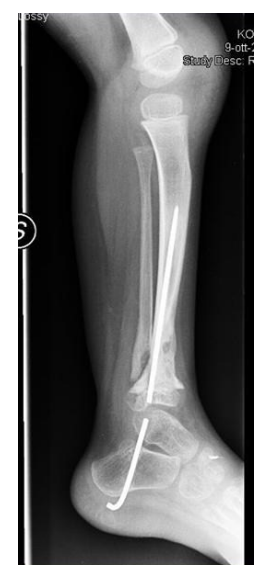

(e)

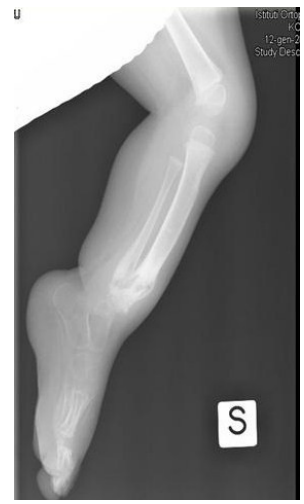

(b)

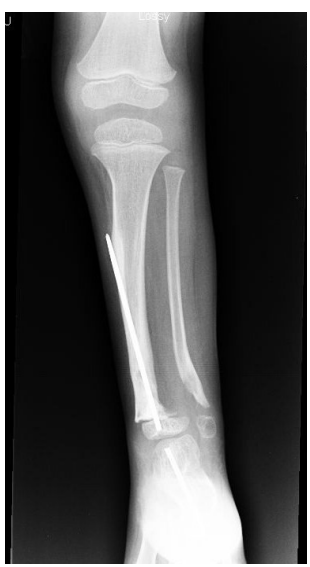

(f)

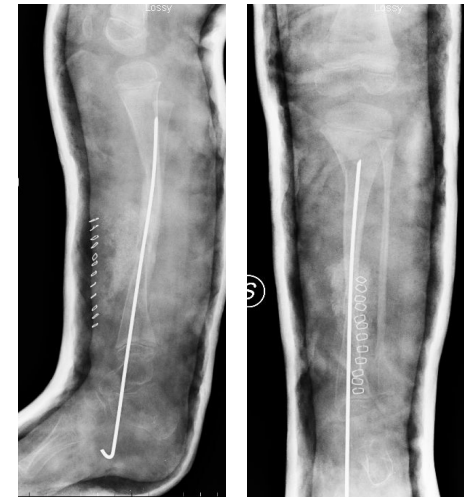

(c)

(d)

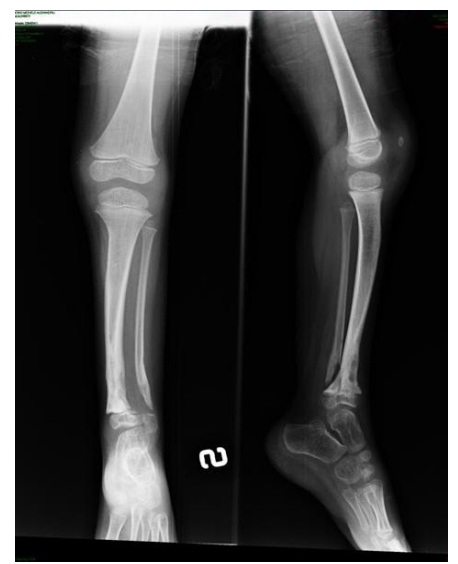

(g)

Figure 2. (a) (b) Pseudoarthrosis non in NF1; (c) (d) Rx after surgical treatment; (e) (f) (g) $\mathrm{XR}$ at follow up at six months nail broken under the lesion and helead pseudoattrosis. 
Case two: Patient male thirteen years old at the time of surgery with tibial pseudoarthrosis in NF1, he had several unsuccesfull surgery before this treatment, he was treated with intramedullary nailing and MSCs and only after three months from surgery there were the bone healing of the lesion we have seen high levels of fibroblastic grow factor in this patient may bee influenting the healing of the lesion that was obtained after tree months (Figures 3(a)-(h)).

Case three: Patient male thirteen years old at the time of surgery with tibial pseudoarthrosis in NF1, he had three surgical procedures before (he had an external fixator and for two times an intramedullary nailing). At the time of surgery he had a distal tibial pseudoarthrosis with a big leg lengthening discrepancy so that we decide to use an external circular fixator according the Ilizarov technique to perform the leg lengthening and to fix the pseudoarthrosis. At seven months from surgery we have seen the distal tibial pseudoarthrosis (Figures 4 (a)-(f)).

After seven months he had a new pseudaorthrosis in the site of bone compactotomy on the proximal metafisis of tibia. All these patients had a distal tibial pseudarthrosis.

Poor results: Eight cases, five male (one of these patient had a pseudoarthrosis in DOFOL bone long osteodisplasia, two patients had a pseudoarthrosis in NF1, and one patient had an idiopatic pseudoarthrosis) and three female, (one with osteodisplasia and two with NF1) two patient lost from follow-up. All the poor the result were the non-union of the bone lesion. In two cases we had a new fracture with broken nail barcking off. In one case we had a deep infection of the bone so that we take off the nail and put a long bone cast and perform an antibiotic therapy for three months.

In the others five cases we didn't see the bone healing but the surgical synthesis was stable and the patient had put on a plaster to walk.

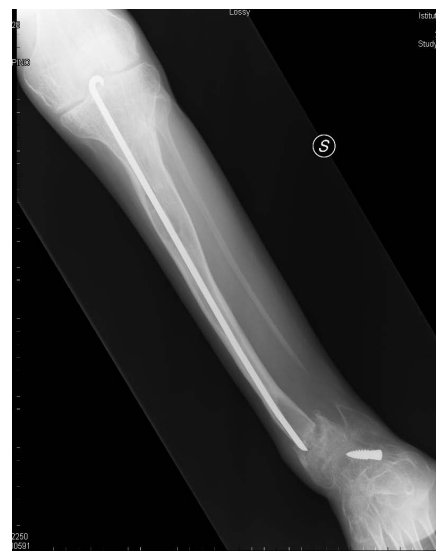

(a)

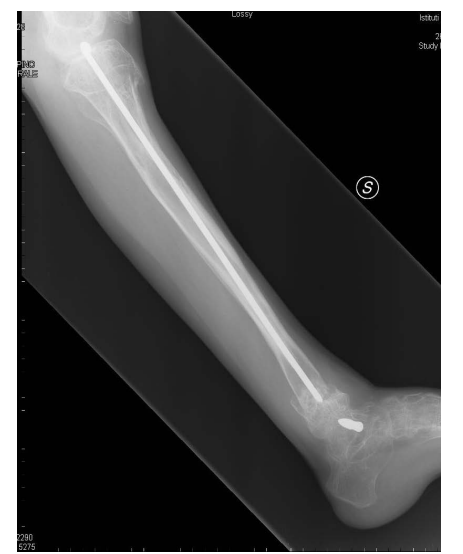

(b)

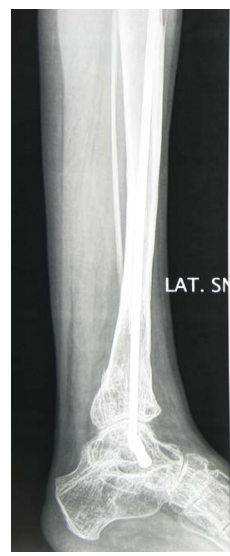

(c)

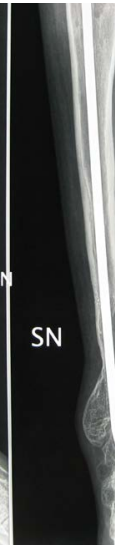

(d)

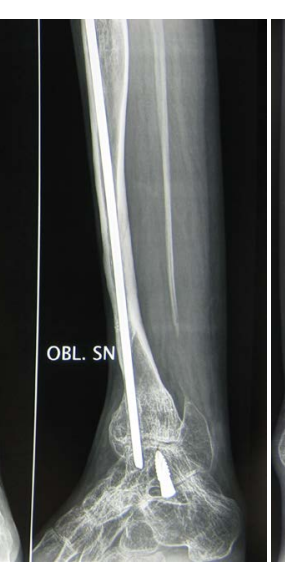

(e)

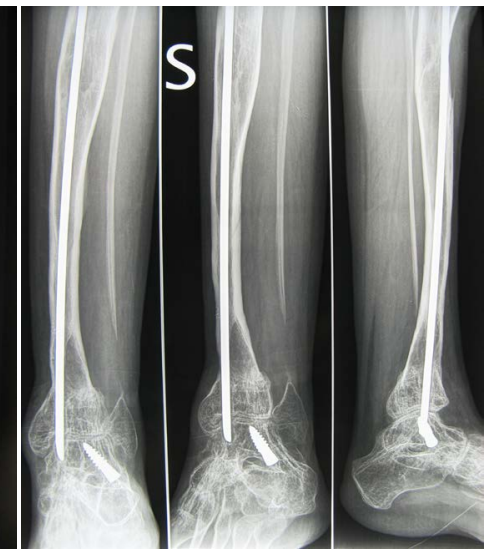

(f) (g) (h)

Figure 3. (a) (b) Pseudoartrosis in NF1 a boy after several operations; (c)-(e) After three months from MSC implantation and surgical synthesis with nail healing of the lesion; (f)-(h) After six months of follow up. 


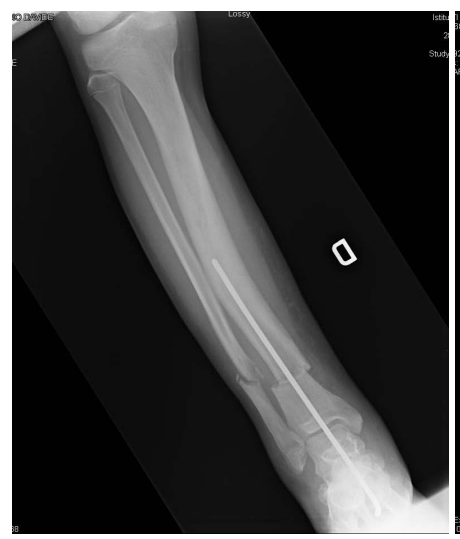

(a)

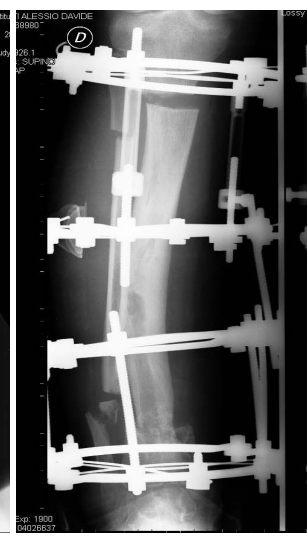

(b)

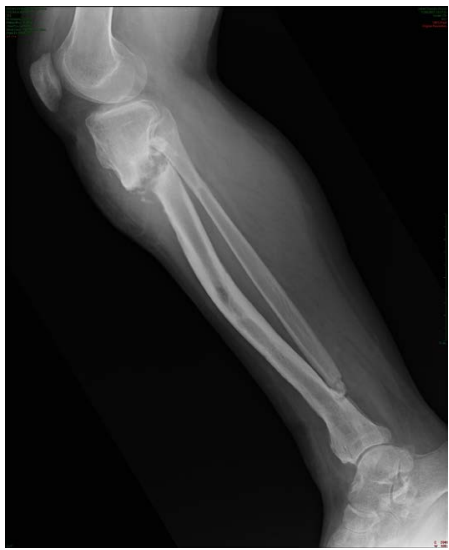

(e)

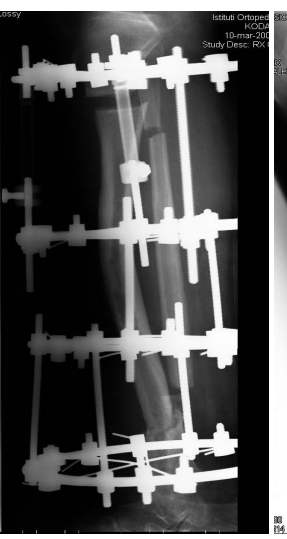

(c)

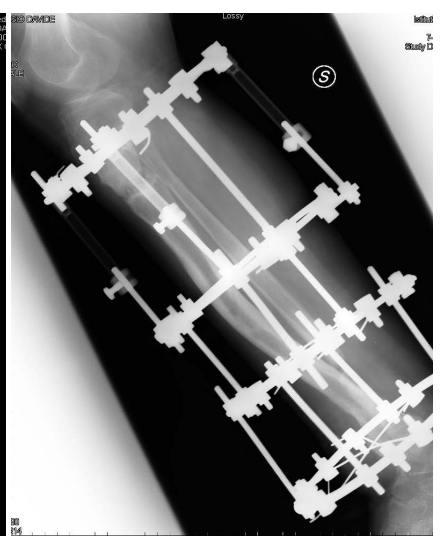

(d)

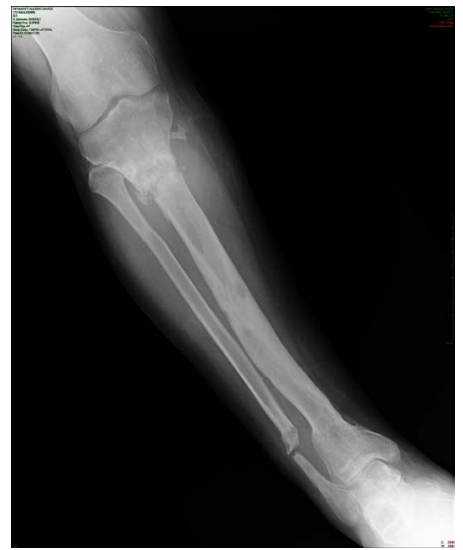

(f)

Figure 4. (a) (b) (c) (d) A boy with pseudoartrhosis and NF1 treated with nailing without healing. The same case treated with circular esxternal fixator and MSC implant; (e) (f) After three months healing of the primitive lesion in the distal tibia and presence of a new lesion on the prosximal tibia.

\section{Discussion}

The biological bases of healing show that MSC (mesenchimal stem cells) transplantation derived from bone marrow resident in iliac crest (IC) were able to differentiate into osteoblast much better than resident in proximity of the pseudoarthrosis site.

Recently the use of autologous MSC has garanted great interest because the biological properties of MSCs candidate them as a good source of osteogenic precursor for bone repair and regeneration.

The ability to generate bone forming cells was higher in (IC-MSC) mesenchimal stem cells form iliac crest than in pseudoarthrosis site (P-MSC).

Others factors as autologous serum and microenvironment could impair the osteogenic differentiation of IC MSC (bibliografia).

All the healing patient are NF1+ and we found in these patients that osteogenic potential of MSC derived from the iliac crest was more than that derived from the affected site: These results have been confirmed by Leskela [2].

However, on the feasibility of this approach non data are still available on the osteogenic potential of MSC transplanted because also the microenvironment properties could impair the successful procedure.

Also platelet-rich plasma has been shown to stimulate osteoblast proliferation in vitro [2].

Probably because the high levels of grow factors that can improve the bone marrow stromal cells to form osteogenic and angiogenetic tissue [8] [9].

The enhanced bone formation might indirectly depend on vascularization; in fact the entire healed patient had a distal tibial pseudoarthrosis. 
The distal tibia has naturally a better vascolarization than the proximal site and in this site.

An increased vascularization achieved also by exogenous delivery of angiogenic growth factor by platelets and by MSC implant contributes to improve the bone formation and consolidation [8].

The improved vascularity might provide better nutrition and increasing resumption and substitution by healthy tissue.

Cell therapy for bone regeneration with lyophilized bone supplemented with platelet gel and bone marrow stromal cells my be an adjuvant in the treatment of congenital pseudoarthrosis of the tibial in children.

We believe that this kind of therapy has a good clinical application because both components are autologous, and easy to perform, it could short the healing time and reduce the number of surgical treatment, especially in little children the use of this combined technique may induce early the bone healing and preserve the articular stifness due to several surgical procedure, and also reduce the leg's discrepancy that is due to the repetite fractures [9] [10].

For all these reasons we believe that is reasonable use this technique also in patient treated with several operations before a demolite surgery or a much more indaginous surgery like vascularized fibula.

\section{References}

[1] Boero, S., Catagni, M., Donzelli, O., Facchini, R. and Freudiani, V. (1997) Congenital Pseudoarthrosis of the Tibia Associated with Neurofibromatosis-1: Treatment with Ilizarov's Device. Journal of Pediatric Orthopaedics, 17, 675684. http://dx.doi.org/10.1097/01241398-199709000-00019

[2] Leskelä, H.V., Kuorilehto, T., Risteli, J., Koivunen, J., Nissinen, M., Peltonen, S., Kinnunen, P., Messiaen, L. and Lehenkari, P. (2009) Congenital Pseudarthrosis of Neurofibromatosis Type 1: Impaired Osteoblast Differentiation and Function and Altered NF1 Gene Expression. Bone, 44, 243-250. http://dx.doi.org/10.1016/j.bone.2008.10.050

[3] Johnston II, C.E. (2002) Congenital Pseudoarthrosis of the Tibia: Results of Technical Variations in the Charnley-Williams Procedure. Journal of Bone and Joint Surgery, 84, 1799-1810.

[4] Rust, P.A., Kalsi, P. and Briges, T.W. (2007) Regulation of Human Stem Cells Differensation into Osteoblasts on Allograft? Clinical Orthopaedics and Related Research, 457, 220-226.

[5] Abdakkah, B.M., Jensen, C.H., Leslie, R.G.Q., Jensen, T.G. and Kassem, M. (2004) Regulation of Human Skeletal Stem Cells Differentiation by D1K1/Pref-1. Journal of Bone and Mineral Research, 19, 841-852.

[6] De Girolamo, L., Sartori, M.F., Arrigoni, E., Rimondini, L., Albisetti, W., Weinstein, R.L. and Brini, A.T. (2008) Human Adipose-Derived Stem Cells as Future Tools in Tissue Regeneration: Osteogenic Differentiation and Cell-Scaffold Interaction. Regenerative Medicine, The International Journal of Artificial Organs, 31, 467-479.

[7] Granchi, D., Devescovi, V., Baglio, S.R., Magnani, M., Donzelli, O. and Baldini, N. (2012) A Regenerative Approach for Bone Repair in Congenital Pseudarthrosis of the Tibia Associated or Not Associated with Type 1 Neurofibromatosis: Correlation between Laboratory Findings and Clinical Outcome. Cytotherapy, 14, 306-314. http://dx.doi.org/10.3109/14653249.2011.627916

[8] Kakudo, N., Shimotsuma, A. and Miyake, S. (2007) Bone Tissue Engineering Using Human Adipose-Derived Stem Cells and Honeycomb Collagen Scaffold. Kansai Medical University, Osaka.

[9] Johnston, C.E. and Birch, J.G. (2008) A Tale of Two Tibias: A Review of Treatment Options for Congenital Pseudoarthrosis of the Tibia. Journal of Children's Orthopaedics, 2, 133-149. http://dx.doi.org/10.1007/s11832-008-0084-2

[10] Erni, D., de Kerviler, S., Hertel, R. and Slongo, T. (2009) Vascularised Fibula Graft for Early Tibia Reconstruction in Infants with Congenital Pseudoarthrosis. Journal of Plastic, Reconstructive Aestetic Surgery, 20, 1-6. 
Scientific Research Publishing (SCIRP) is one of the largest Open Access journal publishers. It is currently publishing more than 200 open access, online, peer-reviewed journals covering a wide range of academic disciplines. SCIRP serves the worldwide academic communities and contributes to the progress and application of science with its publication.

Other selected journals from SCIRP are listed as below. Submit your manuscript to us via either submit@scirp.org or Online Submission Portal.
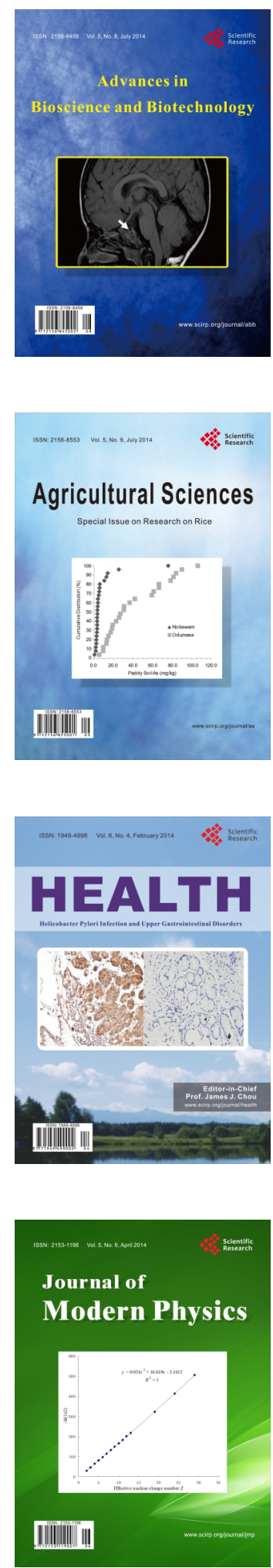
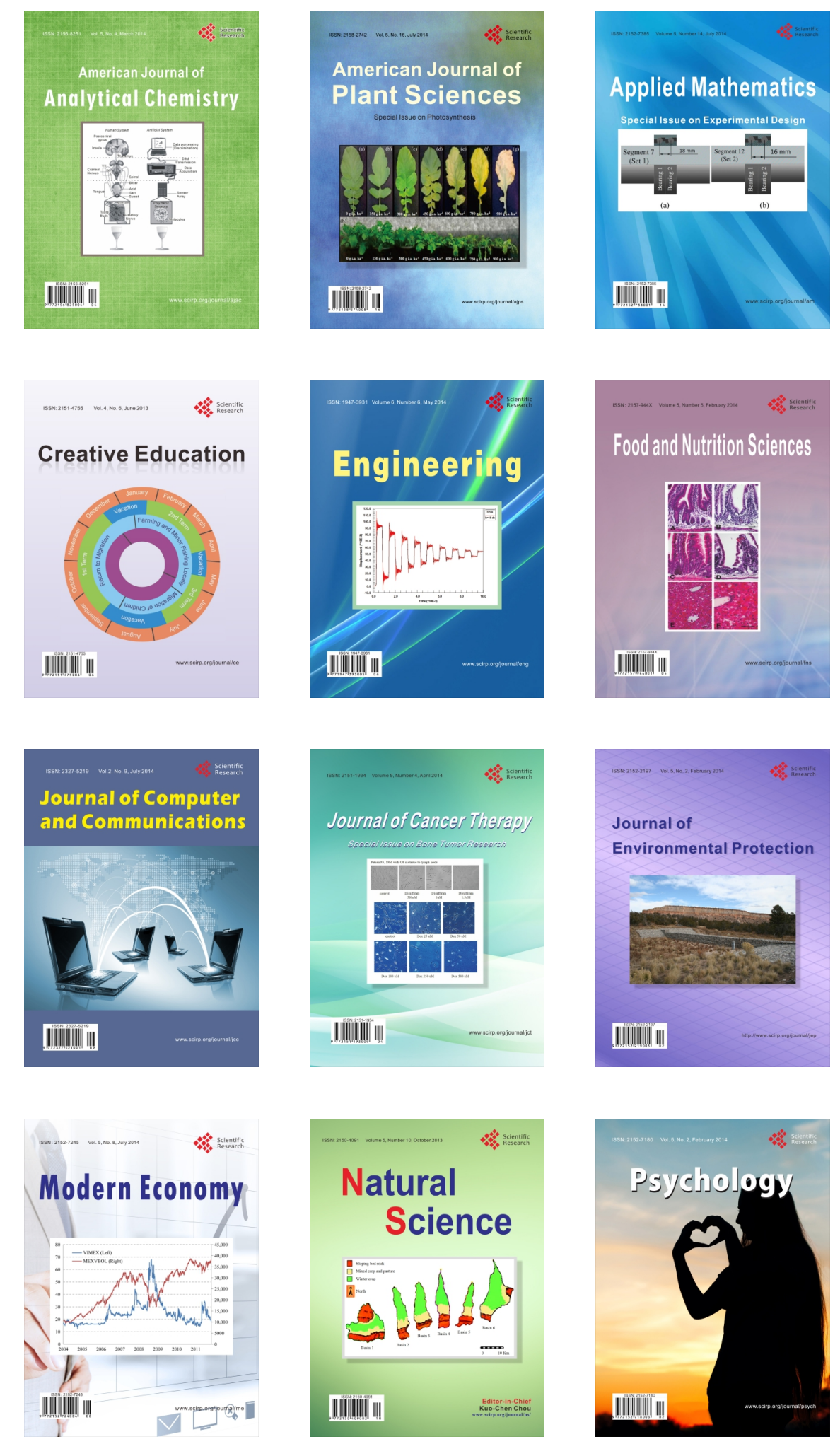